\title{
Prevención de Sintomatología Postraumática en Mujeres con Cáncer de Mama: Un Modelo de Intervención Narrativo
}

\section{Prevention of Posttraumatic Symptomatology in Women with Breast Cancer: A Narrative Intervention Model}

\author{
Felipe E. García \\ Universidad de Concepción y Universidad Pedro de Valdivia, Chile \\ $\&$ \\ Paulina P. Rincón \\ Universidad de Concepción, Chile
}

(Rec: 30 de Noviembre de 2009 / Acep: 10 de Julio de 2011)

\begin{abstract}
Resumen
Esta investigación evalúa un programa grupal narrativo para la prevención de sintomatología postraumática y otras variables asociadas en mujeres recientemente diagnosticadas con cáncer de mama. Para ello se seleccionó a 32 mujeres diagnosticadas en los Hospitales Higueras de Talcahuano y Regional de Concepción; 14 de ellas fueron asignadas al grupo experimental y 18 al grupo control. Los resultados indican que el programa fue efectivo en la reducción de la sintomatología postraumática, sintomatología depresiva y sintomatología somática. No se encontraron diferencias significativas en la variable autoestima. Los puntajes obtenidos en sintomatología postraumática seguían descendiendo a los tres meses de seguimiento. Se analizan alcances y limitaciones de este estudio.
\end{abstract}

Palabras clave: Cáncer de mama, terapia narrativa, estrés postraumático.

\begin{abstract}
This study evaluates a group program narrative for the prevention of post-traumatic symptomatology and other associate variables in women recently diagnosed with breast cancer. 32 women diagnosed with this pathology in two Hospitals: Higueras of Talcahuano and Regional of Concepción were selected; 14 of them were assigned to experimental group and 18 to the control group. Results indicate that the program was effective in the reduction of the post-traumatic, depressive and somatic symptomatology. There were not significant differences in the self-esteem variable. The scores obtained in post-traumatic symptomatology continued descending three months follow up. The reaches and limitations of this study are analyzed.

Key words: Breast cancer, narrative therapy, post-traumatic stress disorder.
\end{abstract}

* Correspondencia: Felipe E. García, Escuela de Psicología, Universidad de Concepción, Ciudad Universitaria s/n, Concepción, Chile.

E-mail:fgarciam@udec.cl 


\section{Introducción}

Según el Instituto Nacional de Estadística (2006), el cáncer ocupa el segundo lugar como causa de muerte en Chile. El cáncer de mama en 2008 alcanzó una tasa de mortalidad observada de 14,5 por 100.000 mujeres. La tasa de Años de Vida Potenciales Perdidos por cáncer de mama en la mujer es de 100 por 100.000 , ocupando el segundo lugar después de cáncer cervicouterino (Ministerio de Salud, 2010).

Gracias a los avances de la tecnología y la medicina, los tratamientos para el cáncer de mama están siendo cada vez más efectivos, prolongando la vida de las personas, disminuyendo su sufrimiento e incluso provocando una remisión total. Por este motivo, la eficacia del tratamiento de cáncer se mide actualmente no sólo por el período de remisión y el tiempo de supervivencia, sino también por la calidad de vida del paciente, su funcionamiento social y familiar, y el grado de ausencia de malestar físico y psicológico (Barroilhet, Forjaz \& Garrido, 2005).

Hablar de cáncer significa enfrentarse al sufrimiento, deterioro, muerte y trascendencia (Barroilhet et al., 2005); su diagnóstico es considerado como un suceso vital estresante que afecta no sólo al individuo que lo recibe, sino también a su entorno familiar y social (Cano, 2005). La adaptación para quien tiene o tuvo cáncer es multidimensional, implica características emocionales, cognitivas, conductuales, físicas, sociales y existenciales (Stanton, Collins \& Sworowski, 2001). Barroilhet et al. (2005) afirman que la adaptación exitosa ocurre en los pacientes que son capaces de reducir el impacto de la enfermedad, regulando el malestar emocional y manteniéndose implicados en los aspectos de la vida que aún están revestidos de significado e importancia para ellos.

El cáncer como trauma, según Petersen, Bull, Propst, Dettinger y Detwiler (2005), amenaza el sentido de cohesión del pasado, presente y futuro del paciente, lo que puede advertirse al escuchar el relato de su experiencia. Esto es consistente con planteamientos generales acerca de creencias básicas que se rompen tras experiencias traumáticas, creencias acerca de invulnerabilidad personal, de sentido de la vida y de que los sucesos son todos ordenados, predecibles y controlables (Sassón, 2004).

Las secuelas psicológicas que más se observan en pacientes con cáncer son los síntomas de ansiedad, depresión y malestar psicológico (Cano, 2005; Maté, Hollenstein \& Gil, 2004). Asimismo, los procedimientos médicos utilizados -mastectomía y quimioterapia-, generan gran variedad de efectos psicológicos en las pacientes (Barra, Hidalgo, Muñoz, Reyes \& Ubilla, 2008; Cruzado, 2006; Olivares, 2007; Rojas-May, 2006), constituyendo experiencias traumáticas para muchas de ellas (Olivares, 2007). Un agravante de estas secuelas es el miedo a la recurrencia de la enfermedad, que acompaña a los supervivientes por largos períodos de su vida, incluso cuando la enfermedad ya ha sido derrotada (Rodríguez, Priede, Maeso, Arranz \& Palao, 2011).
Debido a la alta relación encontrada entre el Trastorno de estrés postraumático (TEP) observado tras el diagnóstico de cáncer (Nordin, Berglund, Glimelius \& Sjoden, 2001; Koopman et al., 2002), se hace necesario intervenir tempranamente para prevenir su desarrollo. La mayoría de las publicaciones sobre intervención en TEP se han centrado en evaluar las intervenciones cuando ya existe diagnóstico (Guerra, Fuenzalida \& Hernández, 2009; Vallejo, 2011; Vallejo \& Terranova, 2009), pero no en desarrollar estrategias preventivas cuando el evento traumático es reciente, aun cuando es posible encontrar revisiones (Echeburúa, 2010) y propuestas de prevención (García \& Mardones, 2010), relacionadas estas últimas con desastres naturales.

Investigaciones en prevención de TEP han evaluado técnicas como el "debriefing", intervención psicosocial breve (Vera, 2005). Sin embargo, metaanálisis realizados por Van Emmerik, Kamphuis, Hulsbosch \& Emmelkamp (2002) y por Aulagnier, Verger \& Rouillon (2004) han encontrado pocos o nulos efectos del debriefing en la prevención del TEP.

Ocurre lo mismo con la consejería psicológica interpersonal (Holmes et al., 2007), que tras seis meses de intervención, menos de la mitad de los participantes se mantenían en el programa y no mostraban diferencias significativas en TEP con el grupo control.

Debido a la insuficiencia de investigaciones acerca de intervenciones que prevengan el TEP en general o en mujeres con cáncer de mama en particular, se han revisado otras investigaciones que han abordado el tema desde lo teórico y que resaltan la importancia específica de la expresión emocional y el apoyo social en la prevención del TEP tanto en la población general como en pacientes con cáncer. La terapia narrativa, desarrollada principalmente por White \& Epston (1993), modelo coherente con los hallazgos de estas últimas investigaciones, ha sido sugerida para la prevención del TEP en esta misma población (Petersen et al., 2005).

Investigaciones guiadas por Pennebaker o realizadas siguiendo sus postulados, han demostrado que la expresión emocional de los significados asociados al trauma previene a largo plazo problemas en la salud física (por ejemplo, los pacientes visitaron menos al médico, mejoró la función inmunológica, redujo la presión sanguínea y el ritmo cardiaco) y psicológica (si bien perciben al principio malestar y tristeza, a largo plazo los síntomas depresivos, la rumiación y la ansiedad general se reducen), entre otras consecuencias (Lacetti, 2007; Owen, Giese-Davis, Córdova, Kronenwetter, Golant \& Spiegel, 2006; Pennebaker, 1994, 1995, 2004; Pennebaker, 1997; Pennebaker, Kiecolt-Glaser \& Glaser, 1988; Pennebaker \& Seagal, 1999; Petrie, Booth \& Pennebaker, 1998).

Barra et al. (2008) concluyen en su estudio cualitativo sobre la vivencia de mujeres con cáncer de mama, que el apoyo social otorgado por amigos, familiares o personas que han pasado por situaciones similares, fue fundamental 
en el proceso de afrontamiento de la enfermedad. Otras investigaciones han destacado la relevancia del apoyo social de la familia (Arora, Finney, Gustafson, Moser \& Hawkins, 2007; Carrasco \& Cea, 2007; Luszczynska, Mohamed \& Schwarzer, 2006), de la pareja (Tamblay \& Bañados, 2004) y del grupo de pares (Durá \& Hernández, 2003) para hacer frente a secuelas psicológicas y sociales derivadas del cáncer, mejorando el nivel de estrés, el ajuste a la enfermedad y la calidad de vida de la paciente. Destacan las investigaciones de Andrykowsky \& Córdova (1998) y de Lewis et al. (2001), que relacionan bajo apoyo social percibido con presencia de TEP en pacientes con cáncer de mama.

Ante la relevancia de la expresión emocional y del apoyo social, el modelo narrativo, cuyo desarrollo se ha visto influido por las investigaciones de Pennebaker (Galarce, 2003; Tarragona, 2003), podría ser un modelo útil para prevenir síntomas de TEP en estos pacientes. Sin embargo, la juventud del enfoque ha proporcionado menos evidencias sobre sus resultados.

El enfoque narrativo de White \& Epston (Epston, 1994; White, 2002a, 2002b; White \& Epston, 1993) se basa en el supuesto de que las narrativas no representan la identidad y los problemas de las personas; las narrativas son la identidad y los problemas. Carr (1998) señala que los problemas aparecen y se mantienen gracias a las historias opresivas que dominan la vida de las personas. Estos relatos no sólo determinan el significado atribuido a sus vivencias, sino también qué aspectos de la experiencia vivida seleccionan para asignarles un significado. El objetivo de la terapia narrativa es ayudar al cliente a reescribir su vida, incorporando a su relato trozos de la historia que han sido marginados y que constituyen excepciones a la narrativa actual; entonces la persona será capaz de dar un nuevo significado a su vida y proyectar un futuro menos opresivo que el que manifiesta en la actualidad. Asimismo, los nuevos significados cobrarían mayor valor al ser transmitidos y compartidos con la red social que rodea y sostiene al paciente, propiciando, diversas instancias en donde es posible establecer esta conexión, ya sea en forma simbólica (cartas terapéuticas) $o$ en forma directa (foros, encuentros familiares). Todos estos elementos se encuentran implícitos en una propuesta de Petersen et al. (2005) en la cual se sugiere un abordaje narrativo grupal para prevenir síntomas de TEP en pacientes que han sobrevivido al cáncer.

Ochoa et al. (2010) aplicaron un programa grupal a 12 supervivientes de cáncer para facilitar el crecimiento postraumático y reducir sintomatología postraumática; utilizaron facilitación de la expresión emocional y fortalecimiento de las redes de apoyo social, buscando la construcción de nuevas narrativas en los pacientes, obteniendo resultados positivos. Si bien su fin fue terapéutico y no preventivo, los resultados muestran la relevancia y coherencia de las variables expuestas.
En conclusión, parece ser que el enfoque narrativo es un modelo adecuado para prevenir o reducir síntomas de TEP asociados al cáncer, sin embargo, se hace necesario realizar estudios para evaluar su eficacia, diseñando programas individuales y grupales replicables y que mejoren efectivamente la calidad de vida de los pacientes supervivientes del cáncer.

Así, la pregunta de investigación es: ¿Representa el modelo de intervención narrativo un enfoque efectivo para prevenir síntomas de TEP en mujeres diagnosticadas con cáncer de mama?

Los objetivos son: (a) elaborar un programa de intervención grupal, breve y eficaz, basado en un modelo narrativo, para prevenir síntomas de TEP en mujeres diagnosticadas con cáncer de mama, y (b) evaluar su eficacia en la prevención de síntomas de TEP, así como en otras manifestaciones: depresión, baja autoestima y síntomas físicos, a corto y mediano plazo.

\section{Método}

\section{Diseño}

Se utilizó un diseño cuasi experimental de grupos no equivalentes, con medidas repetidas en que sólo en el grupo experimental hubo evaluaciones de seguimiento. La variable independiente fue la intervención psicológica. Las variables dependientes fueron la sintomatología postraumática y sintomatología concomitante: depresiva, somática y autoestima.

\section{Participantes}

El universo estuvo constituido por el total de la población femenina de la provincia de Concepción diagnosticada con cáncer de mama entre el 16 de abril y el 31 de septiembre de 2008 y que fueron atendidas en los hospitales Higueras de Talcahuano $(\mathrm{N}=36)$ y Regional de Concepción $(\mathrm{N}=34)$. Razones por las que algunas de ellas fueron excluidas: a) 10 ya habían iniciado la quimioterapia, b) 8 inubicables, c) 5 por residir fuera de la provincia, d) 4 se negaron a participar del estudio, e) 2 por presentar recidivas (no era su primer diagnóstico), f) 2 presentar cáncer de mama en nivel III y IV (metástasis), y g) 2 por analfabetismo. La muestra estuvo constituida por 37 pacientes quienes fueron sometidas a evaluación pretest, de las cuales 32 permitieron una evaluación postest. Participaron en el programa preventivo grupal un total de 14 mujeres y 18 conformaron el grupo de control. Las 14 mujeres del grupo experimental participaron de la intervención hasta el final de la misma, es decir, no hubo deserción. El seguimiento al grupo experimental se realizó a 11 participantes (dos se encontraban en vacaciones fuera de la zona y una había cambiado domicilio).

Criterios de inclusión fueron: (a) haber sido diagnosticada con cáncer de mama en un plazo no mayor de tres meses, antes de la evaluación pretest, (b) cáncer de mama en 
Tabla 1: Variables sociodemográficas al momento de la evaluación pretest

\begin{tabular}{|c|c|c|}
\hline Variable & & $\mathrm{N}(\%)$ \\
\hline \multirow{4}{*}{ Edad (en años) } & 40 o menos & $4(12,4)$ \\
\hline & $41-50$ & $8(25,0)$ \\
\hline & $51-60$ & $10(31,3)$ \\
\hline & 61 o más & $10(31,3)$ \\
\hline \multirow{7}{*}{ Nivel educativo } & Sin estudios & $1(3,1)$ \\
\hline & E. Básica incompleta & $10(31,3)$ \\
\hline & E. Básica completa & $3(9,4)$ \\
\hline & E. Media incompleta & $7(21,9)$ \\
\hline & E. Media completa & $7(21,9)$ \\
\hline & E. Superiores incompletos & $2(6,3)$ \\
\hline & E. Superiores completos & $2(6,3)$ \\
\hline \multirow{2}{*}{ Ingreso per capita } & $\$ 100.000$ o menos & $21(65,6)$ \\
\hline & \$101.000 o más & $11(34,4)$ \\
\hline \multirow{4}{*}{ Estado civil } & Casada & $18(56,3)$ \\
\hline & Viuda & $4(12,5)$ \\
\hline & Soltera & $5(15,6)$ \\
\hline & Separada o Divorciada & $5(15,6)$ \\
\hline \multirow{2}{*}{ Hijos } & Sí & $31(97,0)$ \\
\hline & No & $1(3,0)$ \\
\hline \multirow{2}{*}{ Centro de salud } & Higueras & $19(59,4)$ \\
\hline & Regional & $13(40,6)$ \\
\hline \multirow{4}{*}{ Intervenciones médicas } & Cirugía conservadora & $15(46,9)$ \\
\hline & Mastectomía & $8(25,0$ \\
\hline & Radioterapia & $2(6,3)$ \\
\hline & Sin tratamiento & $7(21,8)$ \\
\hline
\end{tabular}

los estadios I y II, (c) estar recibiendo tratamiento médico en los hospitales Higueras de Talcahuano o Regional de Concepción, (d) residir en la provincia de Concepción, y (e) aceptar participar en la investigación. Los criterios de exclusión fueron: (a) haber iniciado tratamiento quimioterapéutico, (b) presentar diagnóstico de TEP, (c) haber iniciado o concluido terapia para esta problemática, (d) presentar un problema cognitivo que impida procesar información y (e) presentar analfabetismo.

Debido a la urgencia de realizar la intervención pocas semanas después del diagnóstico y antes de que las pacientes fueran sometidas a quimioterapia, el criterio de asignación al grupo experimental fue ir incorporando a éste a las primeras pacientes que se encontraran disponibles para participar. Las restantes fueron consideradas grupo control en lista de espera para una posterior participación en grupos preventivos fuera del estudio. La no equivalencia de los grupos experimental y control se corrigió a través de la elección de procedimientos estadísticos que permitieran controlar las diferencias iniciales en las variables dependientes.
La media de edad de las participantes fue 54 años (rango 33 a 79). Las características sociodemográficas de las participantes se muestran en la Tabla 1.

\section{Instrumentos}

Se utilizó un cuestionario sociodemográfico de elaboración propia a fin de obtener información que permitiera caracterizar la muestra, seleccionar a las participantes y favorecer el análisis de datos. Para medir cada variable dependiente se utilizaron las siguientes escalas:

Sintomatología Postraumática: Escala de Impacto de Evento-Revisada (IEE-R). Instrumento creado por Horowitz, Wilner \& Álvarez (1979), revisada por Weiss y Marmar (1997) y validado en Chile por Caamaño, Fuentes, Sepúlveda y Valenzuela (2006). En este estudio se modificaron los tiempos verbales de los ítems dejándolos en tiempo presente. La consistencia interna, medida por el coeficiente alfa de Cronbach, fue de 0,89 en el pretest, 0,92 en el postest y 0,76 en el seguimiento, resultados que indican confiabilidad satisfactoria. 
Sintomatología Depresiva: Inventario de Depresión de Beck (BDI). Se empleó la versión BDI-II de Beck, Steer \& Brown (1996), traducida al español por Melipillán, Cova, Rincón \& Valdivia (2008). La puntuación total varía de 0 a 63. En esta investigación, el valor obtenido por alfa de Cronbach fue de 0,90 en el pretest, 0,91 en el postest y 0,89 en el seguimiento, indicando confiabilidad satisfactoria.

Autoestima: Escala de Autoestima de Rosenberg. El rango del cuestionario es de 10 a 40 . En algunos estudios se utiliza como punto de corte 29 (véase Rincón, 2003). En la presente investigación, el valor obtenido por el coeficiente alfa de Cronbach fue de 0,84 en el pretest, 0,88 en el postest y 0,93 en el seguimiento, indicadores de un nivel de confiabilidad satisfactoria.

Sintomatología Somática: Inventario de Síntomas Somáticos de Pennebaker (PILL). Fue adaptado por Vera, Sepúlveda \& Contreras (2006) para su utilización en Chile. En la presente investigación, el valor obtenido por el coeficiente alfa de Cronbach fue de 0,88 en el pretest, 0,90 en el postest y 0,83 en el seguimiento, todos estos resultados indican confiabilidad satisfactoria.

\section{Procedimiento}

Se diseñó el programa de intervención tras la revisión bibliográfica de terapia narrativa, el protocolo individual de Pérez \& Vásquez (2001) para intervención narrativa de pacientes con TEP y la propuesta de taller narrativo grupal de Petersen et al. (2005) para la prevención del TEP en pacientes con cáncer. El programa se enfocó en: expresar sentimientos y pensamientos relacionados con el diagnóstico, revalorizar la experiencia del cáncer, construir nuevos significados respecto a la misma, transmitir los nuevos significados a las respectivas redes de apoyo social y, finalmente, realizar un ritual para promover la integración de la experiencia.

Se realizó una aplicación piloto con una muestra de cuatro pacientes diagnosticadas con cáncer de mama, tras lo cual se realizaron correcciones en el programa preventivo grupal.

Para la captación de la muestra se contactó con las Unidades de Patología Mamaria de los Hospitales Higueras de Talcahuano y Regional de Concepción. Las unidades entregaban periódicamente un listado con los datos de las pacientes recién diagnosticadas, las que eran contactadas telefónicamente y tras aceptar participar de la investigación, se les visitaba en su hogar. La evaluación pretest se realizó previa firma de una carta de consentimiento informado, en que se aseguraba confidencialidad y voluntariedad. Si la paciente cumplía los criterios de inclusión se informaba del programa, para luego establecer la fecha de inicio del taller.

La duración total del programa fue de cuatro sesiones de dos horas cada una, con una periodicidad de dos sesiones semanales. Se realizó en total tres grupos, entre el 25 de julio y el 30 de octubre del año 2008.
Se llevó a cabo evaluación postest, tanto al grupo experimental como al grupo control. Tres meses después de terminada la intervención se realizó seguimiento sólo al grupo experimental.

Se respetaron normas de confidencialidad y voluntariedad de las participantes. Asimismo, ante el dilema ético que representa dejar una parte de la muestra sin participar en el programa, se mantuvo a estas pacientes en lista de espera, mientras se realizaba el programa al primer grupo, tras ello se les ofreció participar en un grupo de idénticas características. Por ese motivo, la evaluación de seguimiento sólo se realizó en el grupo experimental.

\section{Resultados}

El análisis de datos se realizó a través del paquete estadístico SPSS 15.0 para Windows. El nivel máximo de error tipo alfa aceptable fue de un 5\%.

Tras realizar un análisis de normalidad de las variables, se observó que los puntajes obtenidos en las variables dependientes de sintomatología postraumática, sintomatología somática y autoestima tenían una distribución cercana a lo normal tanto en el grupo experimental como en el grupo control, por lo que se optó por analizar los datos con estadísticos paramétricos. En el caso de la sintomatología depresiva, como los puntajes obtenidos mostraban una alta asimetría positiva, se procedió a transformarlos mediante la función raíz cuadrada, lo cual permitió obtener una distribución más cercana a lo normal y utilizar también estadísticos paramétricos.

Después de realizar un estudio de homogeneidad en las variables dependientes antes de la intervención, se observó que los grupos sólo eran homogéneos en sintomatología depresiva, en cambio, en sintomatología postraumática, somática y autoestima, no lo eran. Para controlar el efecto de estas diferencias iniciales, se realizó un análisis de covarianza (ANCOVA).

En la tabla 2 se presentan las medias y las desviaciones estándar obtenidas para cada variable, tanto en la evaluación pretest como en la evaluación postest, en ambos grupos, y en la evaluación de seguimiento sólo para el grupo experimental.

En relación a la sintomatología postraumática, en la condición pretest, al comparar los puntajes en ambos grupos (experimental y control), se observa que el primero obtiene un promedio de 25,36 ( $D E=18,25)$, mientras que el segundo obtiene un promedio de 39,44 ( $D E=13,47)$. En cambio, en la condición postest, el grupo experimental obtiene un promedio de 18,00 $(D E=8,10)$, mientras que el grupo control obtiene un promedio de 37,17 $(D E=19,20)$. Se aplicó un ANCOVA empleando como variable dependiente los puntajes del IEE-R del postest, como variable independiente la participación en el programa preventivo grupal y como 
Tabla 2: Promedio y desviación estándar para los grupos experimental $(N=14)$ y control $(N=18)$ en las mediciones pretest y postest, y seguimiento sólo para grupo experimental $(N=11)$

\begin{tabular}{|c|c|c|c|}
\hline Variable & Medición & $\begin{array}{c}\text { Experimental } \\
\text { M (DE) }\end{array}$ & $\begin{array}{l}\text { Control } \\
\text { M (DE) }\end{array}$ \\
\hline \multirow[t]{3}{*}{ Sintomatología postraumática } & Pre & $25,36(18,25)$ & $39,44(13,47)$ \\
\hline & Post & $18,00(8,10)$ & $37,17(19,20)$ \\
\hline & Seguimiento & $12,45(8,86)$ & \\
\hline \multirow[t]{3}{*}{ Sintomatología depresiva } & Pre & $13,07(12,79)$ & $14,56(10,53)$ \\
\hline & Post & $9,71(8,28)$ & $15,78(12,60)$ \\
\hline & Seguimiento & $6,91(7,91)$ & \\
\hline \multirow[t]{3}{*}{ Autoestima } & Pre & $32,64(5,67)$ & $28,50(5,59)$ \\
\hline & Post & $33,79(6,40)$ & $30,67(5,47)$ \\
\hline & Seguimiento & $35,91(4,76)$ & \\
\hline \multirow[t]{3}{*}{ Sintomatología somática } & Pre & $57,07(18,01)$ & $71,22(20,27)$ \\
\hline & Post & $49,36(13,09)$ & $77,22(20,72)$ \\
\hline & Seguimiento & $48,64(15,27)$ & \\
\hline
\end{tabular}

covariable los puntajes del IEE-R del pretest. Los resultados correspondieron a $F(1,29)=6,131, p=0,019$, e indican que existen diferencias estadísticamente significativas en los puntajes de sintomatología postraumática entre quienes participan y no participan del programa.

En relación a la sintomatología depresiva, en la condición pretest, al comparar los puntajes en ambos grupos (experimental y control), corregidos por la función raíz cuadrada, se observa que el primero obtiene un promedio de $3,08(D E=1,97)$, mientras que el segundo obtiene un promedio de 3,51 $(D E=1,54)$. Los resultados de la aplicación de la prueba $t$ de student indican que no existen diferencias significativas en los puntajes entre grupos, $t(30)=0,702$, $p=0,488$, por lo que resultan equivalentes en esta variable antes de la intervención grupal. En la condición postest, se observa que el grupo experimental obtiene un promedio de $2,81(D E=1,40)$, mientras que el grupo control obtiene un promedio de 3,72 $(D E=1,43)$. Los resultados de la aplicación de la prueba $t$ llevan a concluir que existen diferencias significativas en los puntajes entre ambos grupos tras la intervención grupal $t(30)=1,801, p=0,04$ (unilateral).

En relación a la autoestima, en la condición pretest, al comparar los puntajes en los grupos experimental y control, se observa que el primero obtiene un promedio de 32,64 $(D E=5,67)$, mientras que el segundo obtiene un promedio de 28,50 $(D E=5,59)$. En la condición postest, se observa que el grupo experimental obtiene un promedio de 33,79 $(D E=6,40)$, mientras que el grupo control obtiene un promedio de 30,67 $(D E=5,47)$. Se aplicó un ANCOVA empleando como variable dependiente los puntajes de la escala de autoestima en el postest, como variable independiente la participación en el programa preventivo grupal y como covariable los puntajes de la escala de autoestima en el pretest. Los resultados $F(1,29)=0,160, p=0,69$ indican que no existen diferencias estadísticamente significativas en autoestima entre ambos grupos.

En relación a la sintomatología somática en la condición pretest, al comparar los puntajes en los grupos experimental y control, se observa que el primero obtiene un promedio de 57,07 ( $D E=18,01)$, mientras que el segundo obtiene un promedio de 71,22 $(D E=20,27)$. En la condición postest, se observa que el grupo experimental obtiene un promedio de 49,36 $(D E=13,09)$, mientras que el grupo control obtiene un promedio de 77,22 $(D E=20,72)$. Se aplicó un ANCOVA empleando como variable dependiente los puntajes obtenidos en la escala PILL en el postest, como variable independiente la participación en el programa preventivo grupal y como covariable los puntajes obtenidos en la escala PILL en el pretest. Los resultados correspondieron a $F(1,29)=13,033, \mathrm{p}=0,001$, e indican que existen diferencias estadísticamente significativas en los puntajes de la escala PILL en el postest entre grupo experimental y control.

En el seguimiento, aplicando el estadístico $t$ de student para muestras relacionadas en todas las variables estudiadas, se observa que no existen diferencias significativas en las variables dependientes de sintomatología depresiva, $t(10)=1,139, p=0,140$; autoestima, $t(10)=1,305, p=0,11$, y sintomatología somática, $t(10)=0,131, p=0,45$, respecto a los puntajes obtenidos en el postest. En cambio, en sintomatología postraumática se observa una disminución significativa, $t(10)=2,215, p=0,025$, de los puntajes respecto a la evaluación postest.

La tabla 3 presenta el estadístico de contraste utilizado y la significación para los análisis efectuados tanto en la evaluación de la intervención como en el seguimiento. 
Tabla 3: Estadísticos de contraste y significación

\begin{tabular}{lcc}
\hline & Contraste & Significación \\
\hline S. Postraumática Post GE - S. Postraumática Post GC & $\mathrm{F}(1,29)=6,131$ & $0,019^{*}$ \\
S. Postraumática Post GE - S. Postraumática Seguimiento GE & $\mathrm{t}(10)=2,215$ & $0,025^{*}$ \\
S. Depresiva Post GE - S. Depresiva Post GC & $\mathrm{t}(30)=1,801$ & $0,04 *$ \\
S. Depresiva Post GE - S. Depresiva Seguimiento GE & $\mathrm{t}(10)=1,139$ & 0,14 \\
Autoestima Post GE - Autoestima Post GC & $\mathrm{F}(1,29)=0,160$ & 0,692 \\
Autoestima Post GE - autoestima Seguimiento GE & $\mathrm{t}(10)=1,305$ & 0,11 \\
S. Somática Post GE - S. Somática Post GC & $\mathrm{F}(1,29)=13,033$ & $0,01 *$ \\
S. Somática Post GE - S. Somática Seguimiento GE & $\mathrm{t}(10)=0,131$ & 0,45
\end{tabular}

* Significativo

\section{Discusión}

Si bien se pretendía contar con el mayor número posible de mujeres diagnosticadas con cáncer de mama en las fechas establecidas, se incluyó finalmente a 32 de las 70 mujeres que constituían el universo, $45 \%$ del total. La principal razón por la que muchas no pudieron participar fue la rapidez con la que iniciaban la quimioterapia. Ya en el proyecto se había considerado este factor como un criterio de exclusión para participar del estudio, principalmente por los efectos físicos y psicológicos que genera en las mujeres (Malvezzi, 2004; Sirgo, Gil \& Pérez-Manga, 2000; Tamblay \& Bañados, 2004), que las inhabilitaba temporalmente para participar en el programa propuesto. Por tal motivo, era necesario iniciar el grupo antes de que las pacientes comenzaran la quimioterapia, por lo que se propuso un programa que se desarrolló en sólo dos semanas, logrando, quizás por esa misma brevedad, que no hubiera deserción en los grupos aunque sí hubo inasistencias entre sesiones. Debido a la rapidez con la que había que actuar, la asignación a los grupos fue de carácter voluntario y no aleatorio. Estudios similares, como el realizado por Sebastián, Manos, Bueno \& Mateos (2007), también conforman el grupo experimental con participantes voluntarias, constituyendo el resto el grupo control.

En cuanto a los resultados del programa, su efecto más importante fue en sintomatología postraumática, principal objetivo de la investigación, donde se observaron diferencias significativas entre grupo experimental y control en el postest y en el seguimiento. La explicación se encontraría en el hecho de que el programa fue elaborado a partir de la evidencia científica disponible sobre variables que efectivamente participan en la prevención del TEP, coherentes con una intervención de carácter narrativo: (a) facilitar la expresión emocional de los significados asociados a una experiencia traumática, y (b) vincular a los pacientes con redes de apoyo social.

Resultados similares se observaron en sintomatología depresiva, pues mientras en el postest el grupo control mantenía un nivel de depresión leve, el grupo experimental aparecía con ausencia de depresión, nivel que se mantuvo en el seguimiento.

Respecto a sintomatología somática, es relevante que el grupo experimental haya obtenido puntajes significativamente inferiores que el grupo control en el postest (momento en que la mayoría de las participantes se encontraba en quimioterapia), resultados que se mantienen en el seguimiento. Si como efecto del programa disminuyen las quejas somáticas, es posible que disminuya la demanda de atenciones de salud, tanto durante la fase de quimioterapia como una vez concluido el tratamiento médico, con el consecuente incremento en calidad de vida de estas pacientes así como la disminución en los costos por parte del sistema de salud.

En cuanto a autoestima, se observó que el programa no tuvo efectos ni en el postest ni en el seguimiento. Explicación a esto sería los elevados puntajes de autoestima obtenidos por ambos grupos: promedio de 30 puntos para la muestra total en el pretest y 32 puntos en el postest, así como 35 puntos para el grupo experimental al seguimiento (máximo 40 puntos). Ello implicaría que la autoestima no se vio afectada por el diagnóstico inicial de cáncer en esta muestra. Es probable que el efecto negativo del cáncer de 
mama en la autoestima se manifieste en etapas más avanzadas de la enfermedad o en el post-tratamiento médico (Narváez, Rubiños, Cortés-Funes, Gómez \& García, 2008).

En cuanto a las limitaciones del estudio, se pueden mencionar el pequeño tamaño de la muestra, la falta de aleatorización en la asignación a los grupos, la ausencia de grupo control en el seguimiento y la realización de seguimiento sólo a los tres meses de concluida la intervención.

Respecto a estas limitaciones, es necesario reiterar, sin embargo, que la ausencia de grupo control se debió a razones éticas y que la falta de aleatorización se debió a razones prácticas.

Para futuros estudios, sería recomendable evaluar esta intervención en un contexto individual. Si bien se ha privilegiado la intervención grupal, debido a menor costo de tiempo y dinero, además de posibilitar la conexión de las mujeres con otras participantes en su situación (acaso una de las estrategias más importantes de esta intervención), el formato individual permitiría mayor participación y adherencia en la medida que ya no sería necesario conciliar el horario de varias participantes para iniciar un grupo terapéutico, permitiendo aumentar el tamaño de la muestra. Además sería recomendable contar con seguimientos a más largo plazo.

En conclusión, es necesario considerar que las mujeres que sufren cáncer de mama están sometidas a numerosos estresores que afectan su calidad de vida tanto durante el tratamiento médico como una vez finalizado éste, por lo que se hace necesario generar instancias que ayuden al mejoramiento de su calidad de vida. Se cree que el programa presentado permite avanzar en este objetivo. De momento ha mostrado ser eficaz no sólo para prevenir el desarrollo de sintomatología postraumática en esas mujeres, sino también para reducir sintomatología depresiva y somática, al postest y a los tres meses de seguimiento.

\section{Referencias}

Andrykowsky, M. \& Córdova, M. (1998). Factors associated with PTSD symptoms following treatment for breast cancer: Test of the Andersen Model. Journal of Traumatic Stress, 11, 189-203.

Arora, N., Finney, L., Gustafson, D., Moser, R. \& Hawkins, R. (2007). Perceived helpfulness and impact of social support provided by family, friends, and health care providers to women newly diagnosed with breast cancer. Psycho-Oncology, 16, 474-486.

Aulagnier, M., Verger, P. \& Rouillon, F. (2004). Efficiency of psychological debriefing in preventing post-traumatic stress disorders. Revue d'Épidémiologie et de Santé Publique, 52, 67-79.

Barra, C., Hidalgo, M., Muñoz, C., Reyes, P. \& Ubilla, P. (2008). Vivencia del diagnóstico y tratamiento en mujeres con cáncer de mama y su relación con el autoconcepto. Tesis para optar al grado de Licenciado en Psicología, Facultad de Ciencias Sociales, Universidad San Sebastián, Chile.

Barroilhet, S., Forjaz, M. \& Garrido, E. (2005). Conceptos, teorías y factores psicosociales en la adaptación al cáncer. Actas Españolas de Psiquiatría, 33, 390-397.
Beck, A., Steer, R. \& Brown, G. (1996). Manual for the Beck depression inventory - second edition. San Antonio, TX: The Psychological Corporation.

Caamaño, L., Fuentes, D., Sepúlveda, M. \& Valenzuela, E. (2006). Adaptación y validación de la versión chilena de la escala de impacto de evento - revisada. Tesis para optar al grado de Licenciado en Psicología, Facultad de Psicología, Universidad San Sebastián, Chile.

Cano, A. (2005). Control emocional, estilo represivo de afrontamiento y cáncer: Ansiedad y cáncer. Psicooncología, 2, 71-80.

Carr, A. (1998). Michael White's narrative therapy. Contemporary Family Therapy, 20, 485-503.

Carrasco, P. \& Cea, L. (2007). Construcción de significados asociados a los patrones de interacción familiar en mujeres con cáncer de mama, y su relación con el afrontamiento de la enfermedad. Trabajo para optar al grado de Magíster en Psicología Clínica, Universidad Adolfo Ibáñez, Santiago de Chile.

Cruzado, J. (2006). La formulación clínica en psicooncología: Un caso de depresión, aversión a alimentos y problemas maritales en una paciente de cáncer de mama. Psicooncología, 3, 175-187.

Durá, E. \& Hernández, S. (2003). Los grupos de auto-ayuda como apoyo social en el cáncer de mama. Las asociaciones de mujeres mastectomizadas de España. Boletín de Psicología, 78, 21-39.

Echeburúa, E. (2010). The challenge of posttraumatic stress disorder prevention: How to survive a disaster? Terapia Psicológica, 28, 147-154.

Epston, D. (1994). Obras escogidas. Barcelona: Gedisa.

Galarce, E. (2003). Psicología Narrativa: Una revisión de sus aspectos teóricos y sus alcances terapéuticos. Tesina presentada para optar al Grado de Licenciado en Psicología, Facultad de Ciencias Sociales, Universidad de Belgrano, Buenos Aires, Argentina.

García, F. \& Mardones, R. (2010). Prevención de trastorno postraumático en supervivientes del terremoto de Chile de febrero de 2010: Una propuesta de intervención narrativa. Terapia Psicológica, 28, 85-103.

Guerra, C., Fuenzalida, A. \& Hernández, C. (2009). Efectos de una intervención cognitivo-conductual en el aumento de conductas de autocuidado y disminución del estrés traumático secundario en psicólogos clínicos. Terapia Psicológica, 27, 73-81.

Horowitz, M., Wilner, N. \& Álvarez, W. (1979). Impact of events scale: A measure of subjective stress. Psychosomatic Medicine, 41, 209-218.

Holmes, A., Hodgins, G., Adey, S., Menzel, S., Danne, P. \& Kossmann, T. et al. (2007). Trial of interpersonal counselling after major physical trauma. The Australian and New Zealand Journal of Psychiatry, 41, 926-933.

Instituto Nacional de Estadística (2006). Principales causas de muerte en Chile por regiones 1997-2003. Santiago de Chile: Autor.

Koopman, C., Butler, L., Classen, C., Giese-Davis, J., Morrow, G. \& Westendorf, J. et al. (2002). Traumatic stress symptoms among women with recently diagnosed primary breast cancer. Journal of Traumatic Stress, 15, 277-287.

Laccetti, M. (2007). Expressive writing in women with advanced breast cancer. Oncology Nursing Forum, 34, 1019-1024.

Lewis, J., Manne, S., Duhamel, K., Johnson, S., Bovbjerg, D. \& Currie, V. et al. (2001). Social support, intrusive thoughts, and quality of life in breast cancer survivors. Journal of Behavioral Medicine, 24, 231-245.

Luszczynska, A., Mohamed, N. \& Schwarzer, R. (2005). Self-efficacy and social support predict benefit finding 12 months after cancer surgery: The mediating role of coping strategies. Psychology, Health \& Medicine, 10, 365-375.

Malvezzi, C. (2004). Psicooncología, hipnosis clínica, psicoterapéutica comportamental en psicooncologia: Una mirada hacia los pacientes. 5to. Congreso Virtual de Psiquiatría, Interpsiquis, Universitat de Barcelona, España. Extraído en enero 26, 2009, disponible en http://www. psiquiatria.com/articulos/psicosomatica/15018/

Maté, J., Hollenstein, M. \& Gil, F. (2004). Insomnio, ansiedad y depresión en el paciente oncológico. Psicooncología, 1, 211-230.

Melipillán, R., Cova, F., Rincón P. P. \& Valdivia, M. (2008). Propiedades psicométricas del Inventario de Depresión de Beck-II en adolescentes chilenos. Terapia Psicológica, 26, 59-69.

Ministerio de Salud (2011). Guía Clínica Cáncer de Mama. Santiago de Chile: Autor. 
Narváez, A., Rubiños, C., Cortés-Funes, F., Gómez, R. \& García, A. (2008). Valoración de la eficacia de una terapia grupal cognitivo-conductual en la imagen corporal, autoestima, sexualidad y malestar emocional (ansiedad y depresión) en pacientes de cáncer de mama. Psicooncología, 5, 93-102.

Nordin, K., Berglund, G., Glimelius, B. \& Sjoden, P. (2001). Predicting anxiety and depression among cancer patients: A clinical model. European Journal of Cancer, 37, 376-384.

Olivares, M. (2007). Cirugía mamaria: Aspectos psicológicos. Psicooncología, 4, 447-464.

Ochoa, C., Sumalla, E., Maté, J., Castejón, V., Rodríguez, A., Blanco, I. \& Gil, F. (2010). Psicoterapia positiva grupal en cáncer. Hacia una atención psicosocial integral del superviviente de cáncer. Psicooncología, 7, 7-34.

Owen, J., Giese-Davis, J., Córdova, M., Kronenwetter, C., Golant, M. \& Spiegel, D. (2006). Self-report and linguistic indicators of emotional expression in narratives as predictors of adjustment to cancer. Journal of Behavioral Medicine, 29, 335-345.

Pennebaker, J. (1994). El Arte de Confiar en los Demás. Madrid: Alianza Editorial.

Pennebaker J. (1995). Emotion, Disclosure and Health. Washington, DC: APA Press.

Pennebaker, J. (1997). Writing about emotional experiencies as a therapeutic process. Psychological Science, 8, 162-166.

Pennebaker J. (2004). Writing to Heal. A Guided Journal for Recovering from Trauma and Emotional Upheaval. Oakland, CA: New Harbinger Publications, Inc.

Pennebaker, J., Kiecolt-Glaser, J. \& Glaser, R. (1988). Disclosure of traumas and immune function: Health implications for psychotherapy. Journal of Consulting and Clinical Psychology, 56, 239-245.

Pennebaker, J. \& Seagal, J. (1999). Forming a story: The health benefits of narrative. Journal of Clinical Psychology, 55, 1243-1254.

Pérez, P. \& Vásquez, C. (2001, febrero-marzo). Reconceptualizar la psicología del trauma desde los recursos positivos: Una visión alternativa. 2do. Congreso Virtual de Psiquiatría, Interpsiquis, Universitat de Barcelona, España.

Petersen, S., Bull, C., Propst, O., Dettinger, S. \& Detwiler, L. (2005). Narrative therapy to prevent illness-related stress disorder. Journal of Counseling and Development, 83, 41-47.

Petrie, K., Booth, R. \& Pennebaker, J. (1998). The inmunological effects of thought suppression. Journal of Personality and Social Psychology, $75,1264-1272$.

Rincón, P. (2003). Trastorno de estrés postraumático en mujeres víctimas de violencia doméstica: Evaluación de programas de intervención. Tesis para optar al Grado de Doctor en Psicología, Facultad de Psicología, Universidad Complutense de Madrid, España.
Rodríguez, B., Priede, A., Maeso, A., Arranz, H. \& Palao, A. (2011). Cambios psicológicos e intervenciones basadas en mindfulness para los supervivientes de cáncer. Psicooncología, 8, 7-20.

Rojas-May, G. (2006). Estrategias de intervención psicológica en pacientes con cáncer de mama. Revista Médica de Clínica Las Condes, 17, 194-197.

Sassón, M. (2004). Catástrofes y salud mental. Abordajes teóricos y modalidades de intervención. Tesina para optar al Grado de Licenciado en Psicología, Facultad de Humanidades, Universidad de Belgrano, Argentina.

Sebastián, J., Manos, D., Bueno. M. \& Mateos, N. (2007). Imagen corporal y autoestima en mujeres con cáncer de mama participantes en un programa de intervención psicosocial. Clínica y Salud, 18, 137-161.

Sirgo A., Gil, F. \& Pérez-Manga, G. (2000). Intervención cognitivoconductual en el tratamiento de las náuseas y vómitos asociados a la quimioterapia en pacientes con cáncer de mama. Revista Electrónica de Psicología, 3. Extraído en enero 26, 2009, disponible en http://www. psiquiatria.com/psicologia/vol3num2/art_6.htm

Stanton, A. L., Collins, C. A. \& Sworowski, L. (2001). Adjustment to chronic illness: Theory and research. En A. Baum, T. A. Revenson \& J. E. Singer (Eds.), Handbook of Health Psychology (pp. 387-404). Mahwah, NJ: Erlbaum.

Tamblay, A. \& Bañados, B. (2004). Psicooncología del cáncer de mama. Memoria para optar al Título de Psicólogo, Departamento de Psicología, Facultad de Ciencias Sociales, Universidad de Chile.

Tarragona, M. (2003). Escribir para re-escribir historias y relaciones. Psicoterapia y Familia, 16, 45-54.

Van Emmerik, A., Kamphuis, J., Hulsbosch, A. \& Emmelkamp, P. (2002). Single session debriefing after psychological trauma: A meta-analysis. Lancet, 360, 766-771.

Vallejo, A. (2011). Cambios sintomáticos en policías con estrés postraumático y psicoterapia de grupo. Terapia Psicológica, 29, 13-23.

Vallejo, A. \& Terranova, L. (2009). Estrés postraumático y psicoterapia de grupo en militares. Terapia Psicológica, 27, 103-112.

Vera, B. (2005). Trauma y ventilación emocional. ¿Mito o realidad? Cuadernos de Crisis, 2, 7-13.

Vera, A., Sepúlveda, R. \& Contreras, G. (2006). Auto-reporte de síntomas físicos y correlatos psicosociales en trabajadores de la minería. Ciencia \& Trabajo, 8, 74-78.

Weiss, D. S. \& Marmar, C. R. (1997). The Impact of Event-Revised. In J. P. Wilson \& T. M. Keane (Eds.), Assessing Psychiatric Trauma and PTSD: A handbook for practitioners (pp. 412-428). New York: Guilford Press.

White, M. (2002a). El enfoque narrativo en la experiencia de los terapeutas. Barcelona: Gedisa.

White, M. (2002b). Reescribir la vida. Barcelona: Gedisa.

White, M. \& Epston, D. (1993). Medios narrativos para fines terapéuticos. Barcelona: Gedisa. 